\title{
COPD
}

\section{Gute Bronchodilatation hilft, Patienten körperlich aktiv zu halten}

\begin{abstract}
- Unter den Patienten mit chronischen Erkrankungen bewegen sich COPD-Patienten am wenigsten. Eine Vergleichsstudie zeigte, dass sie lediglich auf 2.000 Schritte täglich kommen - Patienten
\end{abstract}

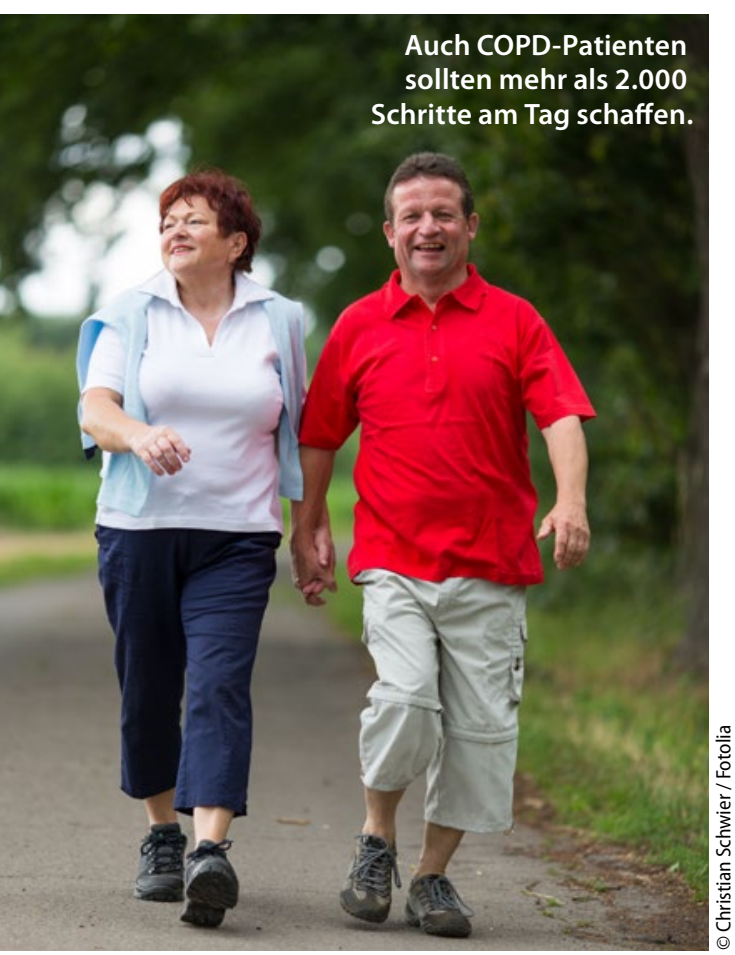

\section{z. B. mit Herzkrankheiten, Diabetes oder} Gelenkrheuma schaffen zwei- bis dreimal so viel, berichtete PD Dr. Hendrik Watz, LungenClinic Großhansdorf.

Inaktivität führt in der Regel zu Muskelatrophie und progredientem Verlust von Lungenfunktion und Belastbarkeit. Deshalb muss man COPD-Patienten zu mehr körperlicher Aktivität zu motivieren, erläuterte Watz. Gesichert ist, dass Lungen-Reha hilft. Muskel-, Ausdauerund Atemtraining und Raucherentwöhnung bessern nachweislich Befinden und Prognose der Patienten.

\section{Zuerst die Lunge entblähen}

Doch muss man laut Watz ,alle Register ziehen“, um die Patienten auch dauerhaft $\mathrm{zu}$ motivieren. Lungensportgruppen sind rar, Watz setzt verstärkt auf Pedometer, Telecoaching, Bewegungsspiele und Smartphone-Apps. Ideal seien auch Gartenpflege oder ein Hund.

Voraussetzung für körperliche Aktivität sei bei COPD-Patienten die Reduktion der Überblähung und Atemnot. Dies gelingt mit dualen Bronchodilatatoren, so Watz. Sie verbessern Lungenfunktion und Belastbarkeit deutlich.
Zur Frage, ob Patienten dadurch tatsächlich aktiver werden, gab es bisher vier Studien: zwei mit negativem, zwei mit positivem Ausgang. Nun präsentierte Watz aktuelle Ergebnisse der MOVE-Studie mit 194 stabilen COPD-Patienten an 30 deutschen Zentren. Sie wurden mit Indacaterol/Glycopyrronium (Ultibro Breezhaler ${ }^{\circledR}$ ) oder Placebo behandelt. Erstmals war ein Aktivitätsparameter einer der primären Endpunkte.

Nach drei Wochen waren die Lungen gut entbläht, die inspiratorische Kapazität der Verumpatienten war um $202 \mathrm{ml}$ besser als jene der Kontrollpatienten. Tatsächlich zeigten sich die Patienten unter Verum auch signifikant aktiver: Sie gingen täglich 358 Schritte mehr und verbrauchten dabei $37 \mathrm{kcal}$ pro Tag mehr als die Kontrollpatienten.

Mit entsprechender Motivation wäre vielleicht mehr drin gewesen. Darauf hat te man in der Studie verzichtet, obwohl Motivation, wie wir gelernt haben, bei COPD der Schlüssel zum Erfolg ist.

Dr. Dirk Einecke

- Presse-Workshop "Mehr bewegen, besser leben-Duale Bronchodilatation punktet als essentieller Baustein im COPD-Therapiemanagement"; Berlin, 28. Januar 2016 (Veranstalter: Novartis)

\section{Erkältungskrankheiten}

\section{Expertengremium empfiehlt Senföle zur kausal orientierten Therapie}

Pflanzliche Senföle aus Kapuzinerkresse und Meerrettich (z. B. enthalten in Angocin ${ }^{\oplus}$ Anti-Infekt N) wirken nachweislich gegen Viren und Bakterien und können aufgrund ihrer multimodalen Wirkweise bei akuten, unkomplizierten Atemwegsinfektionen im Sinne einer kausal orientierten First-Line-Therapie empfohlen werden. Dieses Fazit zogen Ärzte und Wissenschaftler bei einer interdisziplinären Diskussion zu Atemwegsinfektionen in Frankfurt.

„Auf der Suche nach effektiven alternativen Behandlungsansätzen lohnt sich ein Blick in die Natur“, erklärte Prof. Uwe Frank, Freiburg. Wirksamkeit und
Sicherheit der Senföle seien für Erwachsene und Kinder durch mehrere klinische Studien bestätigt worden [z. B. Fintelmann V et al. Curr Med Res Opin 2012;28:1799-807; Goos KH et al. Drug Res 2006;56:249-57; Goos KH et al. Drug Res 2007;57:238-46].

\section{Längerfristig einsetzbar}

In einem Konsensuspapier stellten die Experten fest, dass der Einsatz der Senföle auch bei häufig wiederkehrenden Erkältungskrankheiten sinnvoll ist. Wegen ihrer guten Verträglichkeit seien sie auch wiederholt und längerfristig anwendbar. „Gerade im Anfangsstadium von entzündlichen Atemwegserkrankungen gehören Phytopharmaka zur Therapie der ersten Wahl, denn anders als chemisch-synthetische Antibiotika haben Senföle nicht nur einen einzigen Wirkmechanismus, sondern sie wirken multimodal“, erklärt Prof. Ludger Klimek, Wiesbaden.

Ist eine Antibiotikabehandlung nötig, sollte das Senfölgemisch ergänzend eingesetzt werden, so die Experten. Auch nach Beendigung der antibiotischen Therapie könne es unterstützend wirken und mögliche Rezidive vermeiden.

$$
\text { Red. }
$$

- Nach Informationen von Repha 